\title{
Universal checklist for COVID-19 control measures: consensus via a Delphi method
}

\author{
Authors: Akash Sharma, ${ }^{A}$ Venkatesh $U,{ }^{B}$ Vinh Dong, ${ }^{C}$ Akshay Raut, ${ }^{D}$ Gehad Mohamed Tawfik, ${ }^{E}$ Sze Jia Ng, ${ }^{\mathrm{F}}$ \\ Toka Aziz El Ramly, ${ }^{G}$ Nguyen Hai Nam, ${ }^{H}$ Dmytro Pavlenko, ${ }^{\mathrm{I}}$ Marcel Alied, ${ }^{\mathrm{J}}$ Pierre Elnazir, ${ }^{\mathrm{K}}$ Mahmoud El Marabea, ${ }^{\mathrm{L}}$ \\ Shyam Prakash Dumre, ${ }^{\mathrm{M}}$ Ahmed Elsayad, ${ }^{\mathrm{N}}$ Qusai Alqudah,, ${ }^{\mathrm{O}}$ Mohamed Ahmed Hegab, ${ }^{\mathrm{L}}$ Safia Yahia Zakaria, ${ }^{\mathrm{P}}$ \\ Anh Phuc Nguyet Nguyen, ${ }^{Q}$ Ahmed Sallam ElHawary ${ }^{R}$ and Nguyen Tien Huy ${ }^{S}$
}

\section{Background}

With millions of people infected worldwide, COVID-19 has a high infectivity rate which prompted governments in many countries to launch untested policies and guidelines of unknown impact to contain its spread. We formed an international collaborative group of experts to reach a consensus on a universal checklist that consisted of the most widely implemented containment measures and their timing. This resulting checklist may help further studies to validate the effectiveness of the included measures to ascertain an international response plan against future pandemics.

\section{Methods}

We conducted a two-round modified Delphi study on the expert panel using an email survey. The process of developing the checklist was divided into five phases: planning phase, drafting phase, consensus phase via the Delphi method, dissemination phase, and the maintenance phase.

\section{Results}

An initial checklist consisting of questions about 51 measures was generated after a systematic review and

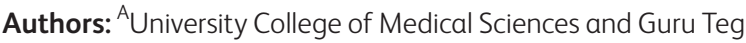
Bahadur Hospital, Delhi, India; ${ }^{B}$ Vardhaman Mahavir Medical College and Safdarjung Hospital, New Delhi, India; ${ }^{C}$ American University of the Caribbean School of Medicine, Cupe Coy, Sint Maarten; ${ }^{D}$ Rajarshee Chhatrapati Shahu Maharaj Government Medical College, Kolhapur, India; ${ }^{E}$ Ain Shams University, Cairo,

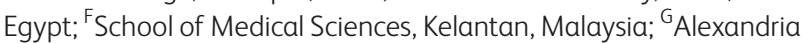
University, Alexandria, Egypt; ${ }^{\mathrm{H}}$ Graduate School of Medicine, Kyoto, Japan; ' ${ }^{Z}$ ir sto protsentiv, Kyiv, Ukraine; 'University of Aleppo, Aleppo, Syria; ${ }^{\mathrm{K}}$ Jagiellonian University Medical College, Krakow, Poland; 'Tanta University, Tanta, Egypt; ${ }^{M}$ Nagasaki University, Nagasaki, Japan; ${ }^{\mathrm{A}} \mathrm{Al}$-Azhar University, Cairo, Egypt; ${ }^{0}$ Jordan University of Science and Technology, Irbid, Jordan; ${ }^{\mathrm{P}}$ Ain Shams University, Cairo, Egypt; ${ }^{Q}$ Concordia College, Moorhead, USA; ${ }^{R}$ South Valley

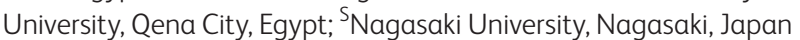

\section{Box 1. Universal checklist of COVID-19 control}

measures

\section{Policy and health system:}

Declaration/announcement of emergency status Launching a public website for guidelines and information Establishing a 'coronavirus task force'/ expert board Conducting training programmes Government financial assistance for citizens/taxpayers Equal protection for immigrants/foreigners

Regulation and stabilisation of food prices and daily necessities Require company to pay full salary to quarantined/isolated people

Financial support to frontline cleaners, toilet attendants and security employees

Free testing

Free treatment

Production/procurement of supplies (such as surgical masks, gloves, ventilators, or goods)

Enhance production of sanitisers

Enhancing hospital capacities (beds)

Designating which hospitals can receive COVID-19 patients Equipping university housings, hotels, sports stadiums or building temporary hospitals to be ready to receive patients Guidelines for treatment of COVID-19

Application of telemedicine

\section{Research:}

Established in-house PCR assay

Development of quick test kits

Successfully identifying SARS-CoV-2 genes

Launching clinical trial treatment of COVID-19

Launching vaccination development

Call for research related to epidemiology, prevention and control measures

Call for development or reuse of efficient low cost of PPE and medical devices such as ventilators

Call for development of a new treatment or new drug discovery, PPE and medical devices such as ventilators Call for non-academic industries to join the research or give funds 


\section{Box 1. Universal checklist of COVID-19 control measures (Continued)}

\section{Social distancing and barriers:}

Prohibition of group gathering more than $(x)$ number of people in public places

Physical distancing from each other in public (2 metres between individuals)

Closure of schools

Working from home

Shelter in place

Closure of public areas

Closure of services

Closure of public transport

Closure of city/area hotspots (separating areas, restriction of movement)

Protection of vulnerable people (elderly, people with suppressed immunities or relevant comorbidities: hypertension, heart disease, kidney disease, liver disease, chronic respiratory disease, diabetes, obesity)

Supporting e-learning for students/tele-workplace

Preventive and containment measures:

Hygiene practice

Wearing face masks in public

Health declaration at airports, seaports and borders

Body temperature and quick test screening at airports,

seaports and borders

Quarantine and prohibition of travellers from affected areas

and other countries

Determining who can get an RT-PCR test

Epidemiological investigation using F0-F5 evaluation system

sent to the experts for rating. Out of the 21 experts who indicated an interest in participating in the study, only 15 completed at least one round of our study and helped create the final checklist. This consisted of 62 measures divided up into the following categories: policy and health system, research, social distancing and barriers, preventive and containment measures, and additional items. The experts were predominantly highly experienced representatives of decision-making healthcare management during COVID-19.

\section{Box 1. Universal checklist of COVID-19 control} measures (Continued)

Using a medical declaration when having respiratory symptoms or close contact with a new confirmed patient Isolation for all confirmed cases (F0)

Disinfect the workplace of the newly detected patient Closure of workplace of the newly detected patient Isolation/quarantine for patients with respiratory symptoms (flu-like illness)

Isolation/quarantine for suspected cases with negative RT-PCR (who had contact with confirmed patients or came from hotspots)

Protection of hospitals at outpatient units

Protection of healthcare workers

Guidelines for each type of health worker to prevent crosstransmission

Guidelines for performing aerosol generation

Guidelines regarding reuse of masks/PPE for healthcare workers

Guidelines on disposal of dead bodies

Guidelines for home care

Guidelines for community service (public transportation, food delivery, postal, volunteer services)

\section{Additional items:}

Triage and dispatch stations outside hospitals

Post lockdown roadmap/strategy: Preparedness of government to lift the lockdown

Isolation/quarantine for suspected cases with negative RT-PCR (who had contact with confirmed patients or came from hotspots)

\section{Conclusion}

A universal checklist of 62 COVID-19 containment measures (Box 1) was developed after meeting experts' consensus via a modified two-round Delphi study with information on what measures they think should be expected during each Centers for Disease Control and Prevention (CDC) stage of the pandemic.

\section{Conflicts of interest}

None declared. 